\title{
Hybrid Heterojunctions of Solution-Processed Semiconducting 2D Transition Metal Dichalcogenides
}

Xiaoyun Yu, ${ }^{\dagger}$ Aiman Rahmanudin, ${ }^{\dagger}$ Xavier A. Jeanbourquin, ${ }^{\dagger}$ Demetra Tsokkou, ${ }^{\ddagger}$ Néstor Guijarro, ${ }^{\dagger}$ Natalie Banerji, ${ }^{\ddagger}$ and Kevin Sivula*,†이

${ }^{\dagger}$ Laboratory for Molecular Engineering of Optoelectronic Nanomaterials, École Polytechnique Fédérale de Lausanne (EPFL), Station 6, 1015 Lausanne, Switzerland

${ }^{\ddagger}$ Department of Chemistry, University of Fribourg, Chemin du Musée 9, CH-1700 Fribourg, Switzerland

Supporting Information

\begin{abstract}
Exfoliated transition metal dichalcogenides (2D-TMDs) are attractive light-harvesting materials for large-area and inexpensive solar energy conversion given their ability to form highly tolerant heterojunctions. However, the preparation of large-area heterojunctions with these materials remains a challenge toward practical devices, and the details of photogenerated charge carrier harvesting are not well established. In this work, we use all solution-based methods to prepare large-area hybrid heterojunction films consisting of exfoliated semiconducting $2 \mathrm{H}-\mathrm{MoS}_{2}$ flakes and a perylene-diimide (PDI) derivative. Hybrid photoelectrodes exhibited a 6-fold improvement in photocurrent compared to that of bare $\mathrm{MoS}_{2}$ or PDI films. Kelvin probe force microscopy, X-ray photoelectron spectroscopy, and transient absorption measurements of the hybrid films indicate the formation of an interfacial dipole at the $\mathrm{MoS}_{2}$ /organic interface and suggest that the photogenerated holes transfer from $\mathrm{MoS}_{2}$ to the PDI. Moreover, performing the same analysis on $\mathrm{MoSe}_{2}$-based hybrid devices confirms the importance of proper valence band alignment for efficient charge transfer and photogenerated carrier collection in TMD/ organic semiconductor hybrid heterojunctions.
\end{abstract}

$\mathrm{T}$ he semiconducting transition metal dichalcogenides (TMDs) have favorable optoelectronic properties for efficient solar energy conversion and, accordingly, have been extensively investigated as promising materials in photoelectrochemical and photovoltaic devices. ${ }^{1}$ Moreover, recent advances leveraging the van der Waals ( $\mathrm{vdW}$ ) layered crystal structure of TMDs, which affords the ability of the material to be exfoliated into $2 \mathrm{D}$ sheets, suggest the possibility of ultrathin flexible and high-performance solar energy conversion devices. ${ }^{2}$ Indeed, single- or few-layer TMDs exhibit superior light-harvesting characteristics compared to their bulk counterparts and can mitigate the disadvantageous anisotropic electronic properties intrinsic to bulk TMDs. ${ }^{3,4}$ Using $\mathrm{p}-\mathrm{n}$ heterojunctions based on only vdW interactions is particularly promising for functional photovoltaic devices as they are exceptionally tolerant to crystal lattice mismatch, affording photogenerated charge separation while enabling low interfacial trap densities. ${ }^{5}$ Accordingly, promising $2 \mathrm{D} \mathrm{p}-\mathrm{n}$ heterojunctions with various semiconducting TMDs or with other nanostructured semiconductors have been recently demonstrated on the micrometer length scale with single exfoliated TMD flakes. ${ }^{6,7}$
Considering hybrid TMD/organic semiconductor (OS) heterojunctions, the tunability of the optoelectronic properties and solution-based processability of organic OSs make them attractive candidates for inexpensive large-area photovoltaics. However, demonstration of large-area hybrid TMD/OS heterojunctions for solar energy applications remains unexplored. Moreover, the fundamental requirements for photogenerated charge separation at the hybrid TMD/OS interface have rarely been described. ${ }^{8-10}$ Thus, developing systems to facilitate the study and optimization of hybrid heterojunctions is an important goal.

In this work, we demonstrate the scalable solution processing of hybrid bilayer heterojunctions based on few-atomic-layer flakes of semiconducting $\mathrm{MoS}_{2}$ or $\mathrm{MoSe}_{2}$ together with an OS. We further scrutinize the nature of the hybrid heterojunction by a number of techniques to establish that the harvesting of photogenerated charge carriers produced by light absorption in 

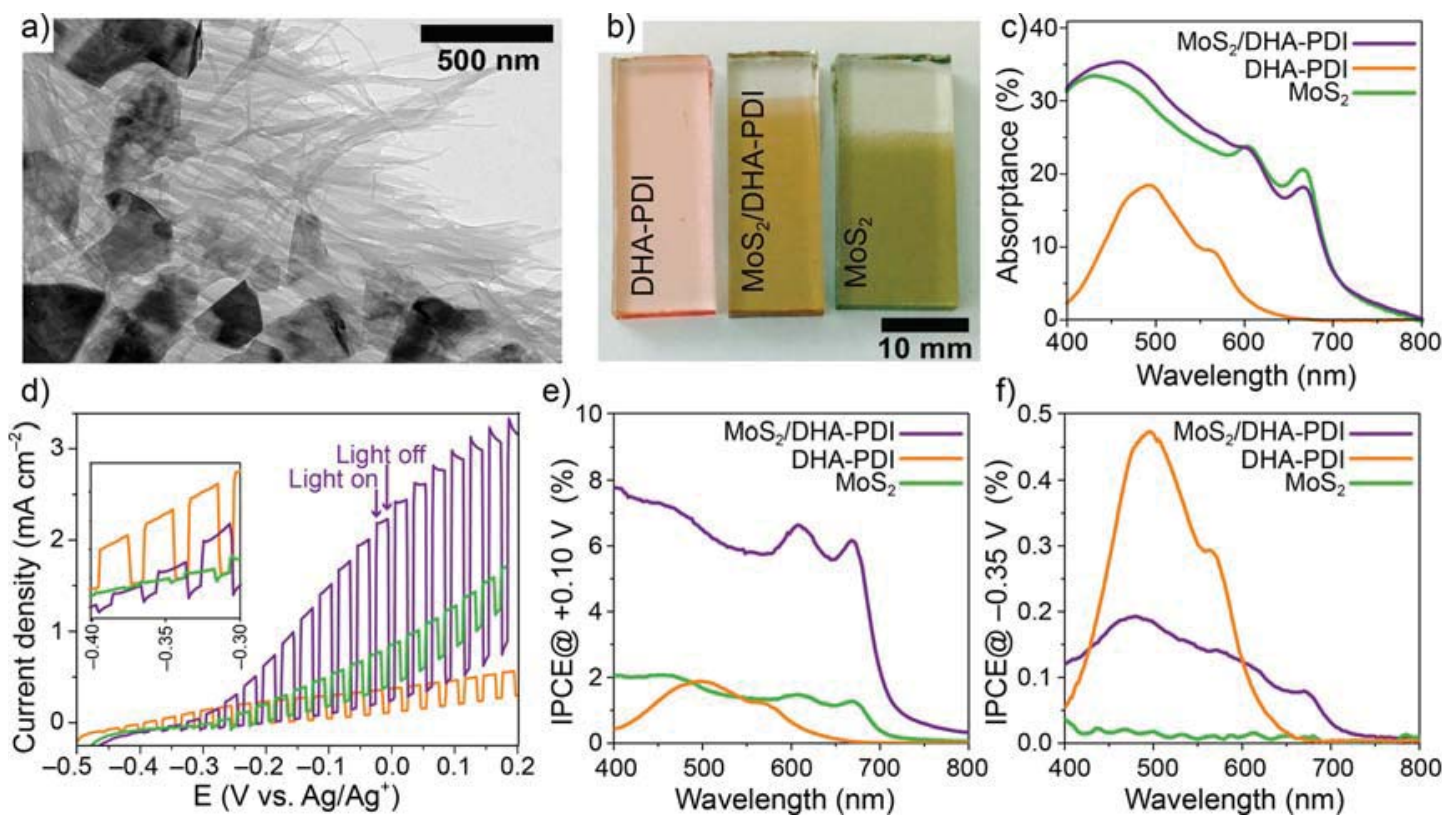

Figure 1. (a) Morphology of the $\mathrm{MoS}_{2}$ /DHA-PDI hybrid bilayer film shown by TEM. (b) photograph of the hybrid and control film deposited on $\mathrm{F} / \mathrm{SnO}_{2}$-coated glass substrates. (c) UV-vis absorptance spectra. (d) $J-V$ curves measured with an $\mathrm{I}^{-} / \mathrm{I}_{3}{ }^{-}$redox couple under intermittent ( 1 sun) illumination and IPCE spectra measured at (e) +0.1 and (f) $-0.35 \mathrm{~V} \mathrm{vs} \mathrm{Ag} / \mathrm{Ag}^{+}$of solution-processed DHA-PDI, $\mathrm{MoS}_{2}$, and $\mathrm{MoS}_{2} /$ DHA-PDI hybrid bilayer electrodes.

the TMD can be greatly enhanced if proper band alignment is present.

First, we exfoliated bulk $\mathrm{MoS}_{2}$ powder in $\mathrm{N}$-methylpyrrolidone $^{11}$ and prepared aggregate-free films using a modified liquid-liquid-based self-assembly method. ${ }^{12}$ The few-layer flakes range in lateral dimension from $\sim 100$ to over $500 \mathrm{~nm}$ and are aligned parallel with the (transparent conducting oxide) substrate surface. A functionalized perylene-diimide (PDI) was chosen as the OS given its good charge transport properties ${ }^{13}$ and planar $\pi$-conjugated core that has been shown to associate with $\mathrm{MoS}_{2}$ via vdW interactions. ${ }^{14}$ Dihexanoic acid (DHA) solubilizing functionalities on the imide position of the PDI were found to be essential for homogeneous film formation of the OS on the $\mathrm{MoS}_{2}$ nanoflake films attributed to the improved wettability. TEM imaging of a bilayer (Figure 1a) shows that DHA-PDI formed a fiber-like morphology ${ }^{15}$ while the films were optically homogeneous over a large area (Figure 1b). Additional morphology characterization and analysis are provided in the Supporting Information (Figures S1-S4). Importantly, the hybrid film showed an identical Raman spectrum (Figure S5) to the bare $\mathrm{MoS}_{2}$ film, implying that only weak noncovalent interactions are present between $\mathrm{MoS}_{2}$ and DHA-PDI. ${ }^{16,17}$

The UV-vis absorptance spectra (Figure 1c) show that the bare $\mathrm{MoS}_{2}$ thin film absorbs $20-30 \%$ of the incident photons from direct gap transitions $(680-400 \mathrm{~nm})$. This value is consistent with an average film thickness of $\sim 10 \mathrm{~nm}$ for $\mathrm{MoS}_{2}$ based on the reported absorption coefficient ${ }^{18,19}$ and an estimated substrate coverage of $80 \%$. The absorptance of the $\mathrm{MoS}_{2}$ /DHA-PDI bilayer hybrid film increases by only about $5 \%$ compared to the bare $\mathrm{MoS}_{2}$ film in the 450-600 nm wavelength range, while a film of only DHA-PDI absorbs up to $18 \%$ of the incident illumination with a peak value located at $500 \mathrm{~nm}$. The different surface properties of the bare substrate and the $\mathrm{MoS}_{2}$ likely result in different amounts of DHA-PDI deposited.
To characterize the optoelectronic performance of the bare $\mathrm{MoS}_{2}$ and hybrid electrodes, thin films prepared on $\mathrm{F}: \mathrm{SnO}_{2}-$ coated glass were analyzed in a three-electrode liquid cell containing the $\mathrm{I}^{-} / \mathrm{I}_{3}^{-}$redox couple (in $\mathrm{MeCN}$ ). This configuration was chosen to facilitate examination of the semiconductor thin films given their small thickness and incomplete coverage, which does not prevent electrical shorting in the solid-state device format. Moreover, nonaqueous electrolyte was selected to eliminate the possibility of $\mathrm{MoS}_{2}$ photocorrosion in water. The $J-V$ curves displayed in Figure 1d illustrate that both the $\mathrm{MoS}_{2}$ and DHA-PDI films alone generated anodic photocurrent under illumination for potentials more positive than -0.35 and $-0.45 \mathrm{~V}$ vs $\mathrm{Ag} / \mathrm{Ag}^{+}$, respectively. The photocurrent density, $J_{\mathrm{ph}}$, was relatively small in both cases, that is, $0.44 \pm 0.04$ and $0.26 \pm 0.05 \mathrm{~mA} \mathrm{~cm}^{-2}$ at $+0.1 \mathrm{~V}$ vs $\mathrm{Ag} / \mathrm{Ag}^{+}$for the $\mathrm{MoS}_{2}$ and DHA-PDI electrodes, respectively, averaging over five replicate electrodes. Remarkably, the hybrid $\mathrm{MoS}_{2}$ /DHA-PDI electrodes gave several times higher $J_{\text {ph }}\left(2.60 \pm 0.29 \mathrm{~mA} \mathrm{~cm}{ }^{-2}\right.$ at $+0.1 \mathrm{~V}$ vs $\left.\mathrm{Ag} / \mathrm{Ag}^{+}\right)$. We note that no significant decay in photocurrent was observed for the hybrid heterojunction electrode during chronoamperometry under intermittent illumination for the minutes times scale (see Figure S6); thus, photocorrosion reactions can be excluded as an origin of the increased photocurrent. This conclusion is further supported by the number of the Mo atoms and PDI molecules present in the electrode, which is $2-3$ orders of magnitude smaller than the number of photogenerated electrons collected during the chronoamperometry measurements.

The incident photon-to-current efficiency (IPCE) spectrum of the $\mathrm{MoS}_{2}$ electrode measured at $+0.1 \mathrm{~V}$ vs $\mathrm{Ag} / \mathrm{Ag}^{+}$(Figure 1e) exhibits the same shape as the thin-film absorption and shows that less than $\sim 2 \%$ of the incident photons can be converted to photocurrent, corresponding to $\sim 7 \%$ absorbed photon-to-current efficiency (APCE) at $670 \mathrm{~nm}$. For the hybrid electrode, the IPCE spectrum shows a similar shape and 

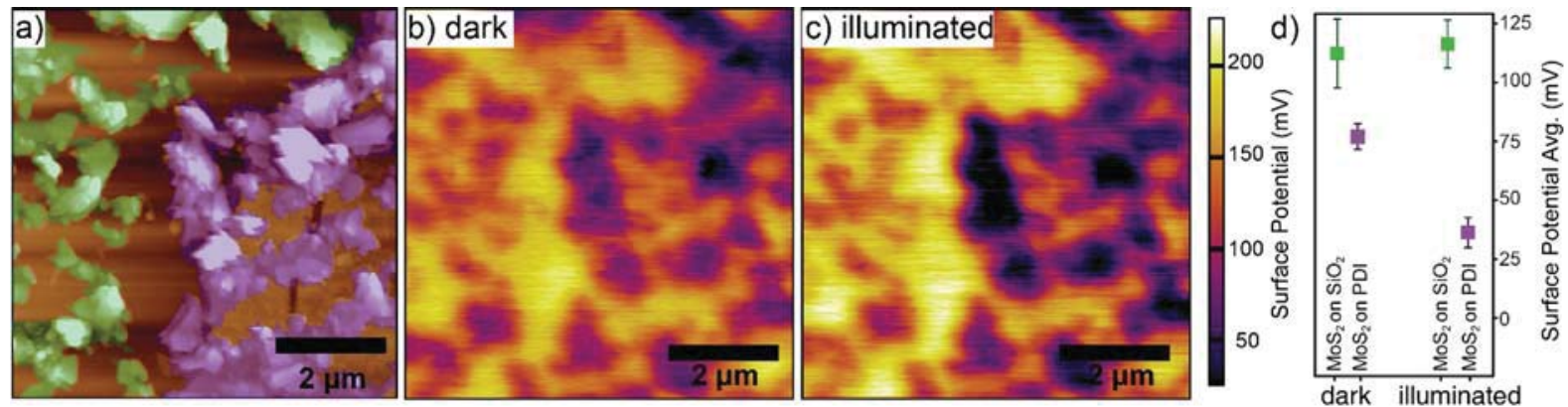

Figure 2. Surface potential measurements. (a) False-colored topography image of $\mathrm{MoS}_{2}$ nanoflakes deposited on bare glass (shaded green) and on a DHA-PDI thin film (shaded purple). The KPFM surface potential maps are given in panel (b) for the dark condition and panel (c) for the illuminated condition, as described in the main text. The average measured surface potential over the flakes for each condition is shown in (d).
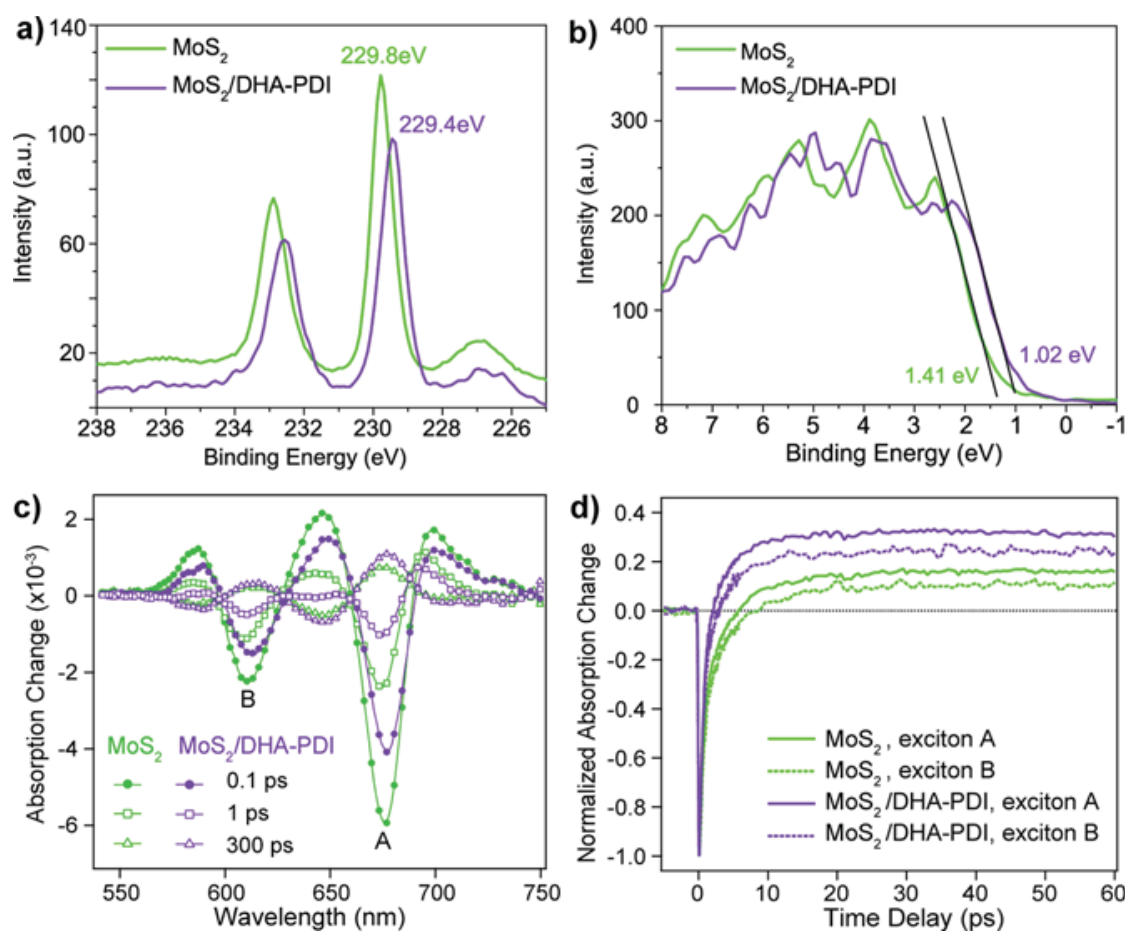

Figure 3. XPS Mo core level (a) and valence band spectra (b) of the $\mathrm{MoS}_{2}$ and $\mathrm{MoS}_{2} / \mathrm{DHA}-\mathrm{PDI}$ hybrid thin films. Transient absorption (TA) spectra (c) of $\mathrm{MoS}_{2}$ and $\mathrm{MoS}_{2} / \mathrm{DHA}$-PDI films recorded at different pump-probe delays; (d) temporal evolution of the normalized absorption change obtained for probe photon energies in resonance with excitonic A $(674 \mathrm{~nm})$ and $B(611 \mathrm{~nm})$ transitions.

dramatic enhancement over the whole $\mathrm{MoS}_{2}$ absorption region compared to the bare $\mathrm{MoS}_{2}$. The APCE at $670 \mathrm{~nm}$ of $\sim 37 \%$ in the hybrid film indicates significantly improved hole extraction from the $\mathrm{MoS}_{2}$ to the redox couple via the DHA-PDI overlayer. The absence of a clear contribution from DHA-PDI absorption in the IPCE of the hybrid bilayer is likely caused by its relatively small light absorption, as seen in the UV-vis spectrum.

Further insight into enhanced charge carrier extraction in the hybrid film was gained from the IPCE spectra measured at $-0.35 \mathrm{~V}$ vs $\mathrm{Ag} / \mathrm{Ag}^{+}$(Figure 1f). At this potential, only a weak driving force is provided for charge separation. Therefore, without DHA-PDI, photogenerated charge carriers in the bare $\mathrm{MoS}_{2}$ electrode recombine before being extracted, leading to the absence of sustained $J_{\mathrm{ph}}$ (see the inset of Figure $1 \mathrm{~d}$ ) and an IPCE below the signal-to-noise detection limit. In the hybrid electrode, we observed an IPCE that originates from photon absorption by $\mathrm{MoS}_{2}$. This suggests that DHA-PDI promotes charge extraction from $\mathrm{MoS}_{2}$ in the hybrid heterojunction. We note that the shape of the hybrid electrode IPCE at $-0.35 \mathrm{~V}$ is slightly different from the IPCE at $+0.1 \mathrm{~V}$ due to the enhanced relative contribution from DHA-PDI with direct contact to the substrate.

The enhancement in photoactivity with the hybrid film was confirmed using two-electrode photovoltaic cells (Figure S7). At 1 sun illumination, short-circuit current density remarkably increased from 0.1 ( $\mathrm{MoS}_{2}$ only) to $0.7 \mathrm{~mA} \mathrm{~cm}{ }^{-2}$ for the hybrid cell, following the same trend of three-eletrode photoelectrochemical results. A clear improvement in open-circuit voltage (from 25 to $80 \mathrm{mV}$ ) was also observed. We note that the small $V_{\mathrm{OC}}$ could reasonably be improved by choosing a redox couple with a more positive oxidation potential, including blocking layers and optimizing the flake size and thickness. However, the drastic increase in photocurrent afforded by the hybrid film is remarkable given previously reported photoperformance of solution-processed TMD semiconductors. ${ }^{20,21}$ 
One possible explanation for the enhancement of $J_{\mathrm{ph}}$ could be the simple suppression of recombination due to the passivation of charge trapping sites on the $\mathrm{MoS}_{2}$. Indeed, the carboxylic functionality is well-known to associate with surface species of many oxides and chalcogenides. This possibility can be addressed by exposing the bare $\mathrm{MoS}_{2}$ film to hexanoic acid, which yielded no improvement (see Figure S8), suggesting that the carboxylic acid functionality does not effectively passivate recombination. Similarly, other possibilities, such as $\mathrm{Cl}^{-}$ion adsorption $^{22}$ or etching ${ }^{23}$ induced by $\mathrm{HCl}$ used in the DHAPDI coating process were excluded by control experiments (Figure S8).

We next employed scanning Kelvin probe force microscopy (KPFM, Figure 2) to map the surface potential of individual $\mathrm{MoS}_{2}$ flakes in contact with either bare glass (green-colored flakes in Figure 2a) or DHA-PDI (purple-colored flakes) during the same scan, afforded by a specially prepared film (see the Supporting Information for a full experimental description). KPFM results acquired in air under dark and illuminated (ca. 0.1 sun) conditions are shown in Figure $2 b-d$. In the dark, the $\mathrm{MoS}_{2}$ flakes can be clearly distinguished as they show a lower surface potential compared to that for DHA-PDI or the substrate. Under illumination, the surface potential of the $\mathrm{MoS}_{2}$ on DHA-PDI decreases by $40 \mathrm{mV}$ on average, while the $\mathrm{MoS}_{2}$ flakes on bare glass remain approximately constant. For further comparison, a surface potential difference map (subtracting light and dark results) is shown in the Supporting Information (Figure S9).

If only defect passivation occurred at the DHA-PDI/ $\mathrm{MoS}_{2}$ interface, no change would be expected in the surface potential difference at these measurement conditions (i.e., in the absence of a built-in potential from conductive contacts or redox species). Instead, the observed surface potential difference between the dark and light conditions implies the accumulation of negative charges ${ }^{24,25}$ in the $2 \mathrm{D} \mathrm{MoS}$ flakes when in contact with DHA-PDI and under illumination. This charging of the 2D flakes is consistent with either the photogenerated charge redistribution in the $\mathrm{MoS}_{2}$ flakes (caused by a dipole induced at the DHA-PDI/ $\mathrm{MoS}_{2}$ interface and the resultant electric field that would drive photogenerated holes to the $\mathrm{MoS}_{2} / \mathrm{PDI}$ interface and electrons away from the interface) or from the direct photogenerated hole transfer from $\mathrm{MoS}_{2}$ flakes to the OS (leading to an accumulation of photogenerated electrons in the $\mathrm{MoS}_{2}$ ).

To gain further insight into the $\mathrm{MoS}_{2}$ /DHA-PDI interface and the possible presence of an interfacial dipole, X-ray photoelectron spectroscopy (XPS) was used, and the spectra for the Mo core level and valence band are shown in Figure 3a,b. First, we note that no signal consistent with $\mathrm{Mo}-\mathrm{O}$ coupling (binding energy higher than $234 \mathrm{eV}$ ) was observed before or after DHA-PDI deposition, consistent with the absence of covalent bonding between $\mathrm{MoS}_{2}$ and DHA-PDI via carboxylic group anchoring or the oxidation of $\mathrm{MoS}_{2}$. The Mo $3 \mathrm{~d}$ peaks are found to uniformly shift $0.4 \mathrm{eV}$ to lower binding energy when the DHA-PDI overlayer is present, which can be attributed to a Fermi level shift caused by chemical doping from an overlayer or to the formation of a built-in potential via interfacial dipole creation. ${ }^{26,27}$ The absence of a clear shift in Raman spectra (Figure S5) after DHA-PDI coating indicates that any chemical doping effect is very weak. Moreover, in Figure $3 \mathrm{~b}$, the valence band spectra with organic coating shows a shift from 1.41 to $1.02 \mathrm{eV}$, equivalent to that observed in the core level spectrum. The consistent shift of $0.4 \mathrm{eV}$, which can be further confirmed by the $S 2 p$ spectra (see Figure S10), clearly indicates the formation of an interfacial dipole, likely a result of an equilibration of the Fermi level of the inorganic with the polaron level of the OS, as described previously in a similar hybrid system. ${ }^{28}$ In our case, an electric field in the $\mathrm{MoS}_{2}$, which would drive free holes toward the interface with the DHA-PDI layer, is reasonably present, consistent with the KPFM results.

The presence of an interfacial dipole that could promote charge separation in $\mathrm{MoS}_{2}$ does not preclude the presence of charge transfer from the $\mathrm{MoS}_{2}$ to DHA-PDI. To gain further insight into the charge carrier dynamics in this system, the bare and hybrid films were measured by femtosecond transient absorption (TA) spectroscopy. The absorption change upon pumping at $600 \mathrm{~nm}$ was probed at different times up to $1 \mathrm{~ns}$ after photoexcitation. The resulting TA spectra for selected time delays are shown in Figure 3c. The complete results and detailed interpretation of the TA spectra are described in the Supporting Information (section 5, Figures S13 and S14). In brief, similar to a previous report, ${ }^{29}$ we observe for both $\mathrm{MoS}_{2}$ and $\mathrm{MoS}_{2}$ /DHA-PDI films negative photobleaching bands from the excitonic states $\mathrm{B}$ and $\mathrm{A}$ of $\mathrm{MoS}_{2}$ at 0.1 ps (centered at 611 and $674 \mathrm{~nm}$, respectively), which decay rapidly (in $300 \mathrm{ps)}$ together with blue shifts to 589 and $647 \mathrm{~nm}$, respectively, owing to the formation of free charge carriers at the direct band edges. ${ }^{29}$ The presence of free carriers at the direct band edges results in positive photoinduced absorption signatures at long times, which decay with identical time constants as the corresponding photobleaching of free charges. As noted in previous work, the photoinduced absorption signatures coincide with wavelengths of the excitonic transitions, allowing quantification of both exciton (short time) and free charge carrier concentration (longer time) using the signal at $674 \mathrm{~nm}$.

Comparing the TA spectra of the $\mathrm{MoS}_{2}$ and $\mathrm{MoS}_{2} / \mathrm{DHA}$ PDI films measured with equivalent pump fluence $(\sim 1 \mu \mathrm{J}$ $\mathrm{cm}^{-2}$ ), the identical features noted at all time delays (Figure $3 \mathrm{c}$ ) indicate that the TA signatures of $\mathrm{MoS}_{2}$ were not significantly perturbed by DHA-PDI (the TA signals for DHA-PDI at the pump wavelength are significantly smaller; see Figure S15). Therefore, a similar number of absorbed photons and initial excitations was generated in both films. However, the TA signal of the hybrid film consists of a smaller amplitude of the photobleaching signals from the excitonic states recorded at early time delays $(t=0.1 \mathrm{ps})$ and a higher amplitude of the free charge signatures at $t=300$ ps compared to those of the $\mathrm{MoS}_{2}$ bare film. The presence of additional long-lived free carriers at the direct band edges in the hybrid film was confirmed by the normalized temporal evolution of the TA signals probed at 674 and $611 \mathrm{~nm}$ (Figure 3d). Further TA measurements with different initial carrier densities (see Figure S16 with increasing excitation fluence to $2 \mu \mathrm{J} \mathrm{cm}^{-2}$ ) or with higher pump photon energy $(3 \mathrm{eV})$ followed the same trend.

The greater free charge carrier concentration in the direct bands in the $\mathrm{MoS}_{2} / \mathrm{DHA}-\mathrm{PDI}$ film implied by the TA results can be rationalized by an increased photogenerated charge carrier separation, which reduces recombination in the PEC measurement. However, this effect could be due to either the interfacial dipole-induced charge separation or hole transfer from the $\mathrm{MoS}_{2}$ to the organic due to exciton splitting at the hybrid junction. We note that because the dynamics of the exciton decay on the few-picosecond time scale are very similar in both case (see Figure S17), the smaller initial concentration of excitons observed after $0.1 \mathrm{ps}$ in the hybrid films could 

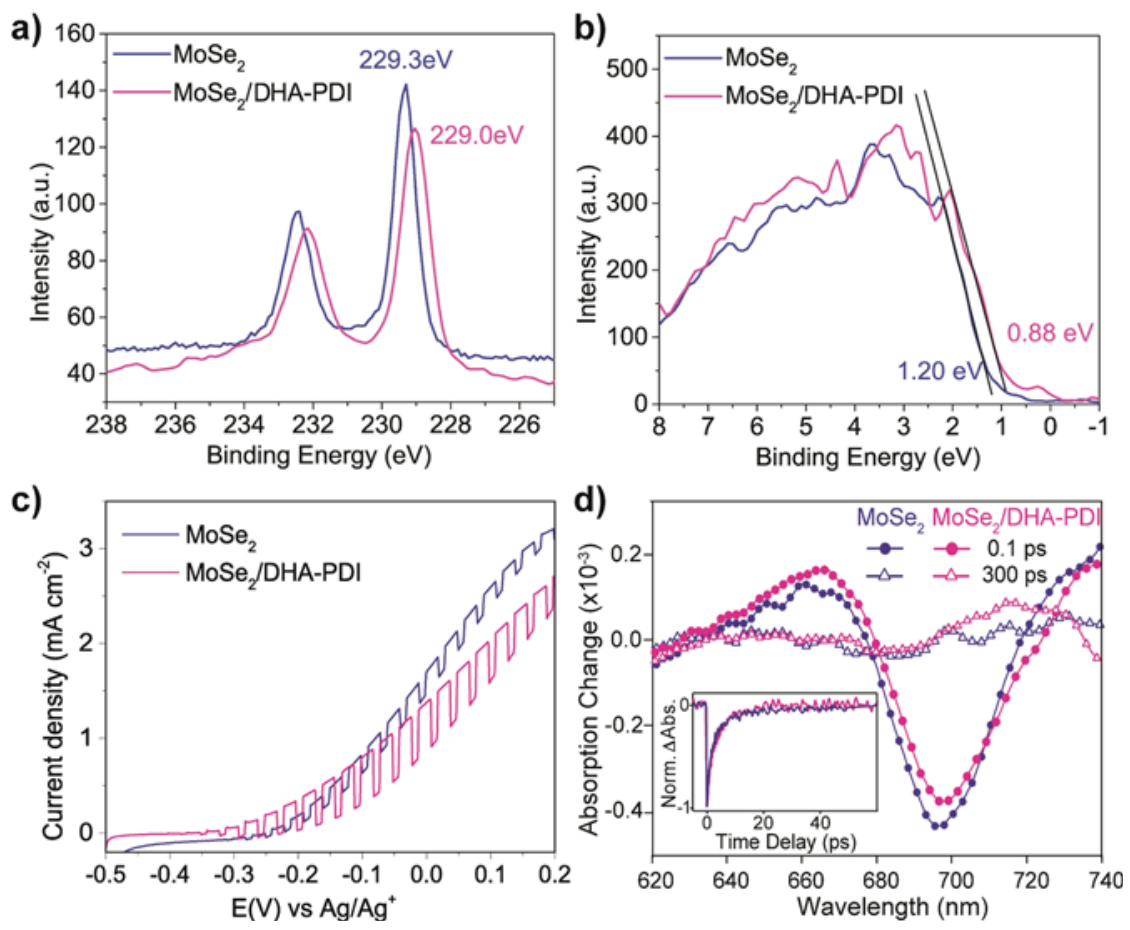

Figure 4. XPS (a) Mo core level and (b) valence band spectra of $\mathrm{MoSe}_{2}$ and $\mathrm{MoSe}_{2} / \mathrm{DHA}-\mathrm{PDI}$ hybrid thin films. (c) $J-V$ curves of MoSe $\mathrm{e}_{2}$ and $\mathrm{MoSe}_{2}$ /DHA-PDI electrodes with $\mathrm{I}^{-} / \mathrm{I}_{3}^{-}$as the redox couple under intermittent $(1$ sun) illumination. (d) TA spectra recorded at pumpprobe delays of 0.1 and $300 \mathrm{ps}$ using excitation at $600 \mathrm{~nm}$ and a fluence of $\sim 1 \mu \mathrm{J} / \mathrm{cm}^{2}$ with (inset) normalized temporal evolution of the absorption change obtained for probe photon energies in resonance with the excitonic $B$ transitions (the A exciton coincides with the fundamental laser wavelength and could not be probed).

possibly suggest that the additional free charge generation in $\mathrm{MoS}_{2}$ in the presence of DHA-PDI occurs faster than our experimental time resolution $(<100 \mathrm{fs})$. Unfortunately, further insight into the possibility of ultrafast charge transfer to organic was not possible due to the weak TA signal from DHA-PDI, which overlaps with the signal from $\mathrm{MoS}_{2}$. Lastly, other possible explanations for the smaller initial exciton concentration in the hybrid film, such as excitation (resonance) energy transfer from the $\mathrm{MoS}_{2}$ to the PDI, can be excluded based on the energy levels.

Because the TA results cannot unambiguously corroborate photogenerated hole transfer from the $\mathrm{MoS}_{2}$ to the PDI, we next hypothesized that interfacial hole transfer would depend on the relative valence band positions of the TMD. To investigate this factor, we fabricated a bilayer film based on the selenium TMD (i.e., $\mathrm{MoSe}_{2}$ /DHA-PDI), given that the valence band edge level of $\mathrm{MoSe}_{2}$ is $\sim 0.5 \mathrm{~V}$ higher in energy than that in $\mathrm{MoS}_{2} \cdot{ }^{30,31}$ Due to the similar crystal structures of $\mathrm{MoS}_{2}$ and $\mathrm{MoSe}_{2}$, the fabrication of $\mathrm{MoSe}_{2}$ and $\mathrm{MoSe}_{2} / \mathrm{DHA}-\mathrm{PDI}$ bilayer films by the same experimental methods and obtaining comparable morphology and similar flake size and thickness (ca. $10 \mathrm{~nm}$ ) were possible.

Similar to the $\mathrm{MoS}_{2}$ /DHA-PDI film, the XPS spectra of the $\mathrm{MoSe}_{2} / \mathrm{DHA}-\mathrm{PDI}$ bilayer thin film (Figure $4 \mathrm{a}, \mathrm{b}$ ) show an equivalent shift of $0.3 \mathrm{eV}$ for both the Mo $3 \mathrm{~d}$ core level and valence band level compared to bare $\mathrm{MoSe}_{2}$, indicating the presence of an interfacial dipole. The $J-V$ curves (Figure 4c) from the photoelectrochemical measurements show similar photocurrent for the bare $\mathrm{MoSe}_{2}$ film compared to that for $\mathrm{MoS}_{2}$. A higher dark current is likely a result of higher catalytic activity of defects and edge sites on $\mathrm{MoSe}_{2}$ flakes. ${ }^{32}$ However, the PEC behavior in the $\mathrm{MoSe}_{2} / \mathrm{DHA}-\mathrm{PDI}$ hybrid photoelectrodes was in stark contrast to that of the $\mathrm{MoS}_{2}$-based hybrid electrodes. The photocurrent increased only slightly from $0.21 \pm 0.03\left(\mathrm{MoSe}_{2}\right.$ only) to $0.37 \pm 0.09 \mathrm{~mA} \mathrm{~cm}$ $\left(\mathrm{MoSe}_{2} / \mathrm{DHA}-\mathrm{PDI}\right)$ at $+0.1 \mathrm{~V}$ vs $\mathrm{Ag} / \mathrm{Ag}^{+}$(average over five electrodes). Moreover, the TA spectra for the $\mathrm{B}$ exciton of the $\mathrm{MoSe}_{2}$ and hybrid bilayer films (Figure 4d) does not indicate significant change in the TA spectra at $0.1 \mathrm{ps}$ (mainly excitonic transitions) or at $300 \mathrm{ps}$ (long-lived free carriers). In addition, the normalized temporal decay of the $\mathrm{B}$ exciton (Figure $4 \mathrm{~d}$ inset) is identical for the bare and hybrid films. These TA results are consistent with the insignificant photocurrent increase observed for the hybrid electrodes in the photoelectrochemical measurements.

Overall, the results with $\mathrm{MoSe}_{2}$ help to complete a clear picture of the important factors leading to the enhanced photocurrent in the $\mathrm{MoS}_{2} / \mathrm{DHA}-\mathrm{PDI}$ films. From the XPS spectra for $\mathrm{MoS}_{2}$ and $\mathrm{MoSe}_{2}$, DHA-PDI coating on both TMDs creates an interfacial dipole, thus implying band bending in the TMD at the hybrid interface. Such band bending potentially provides a driving force for charge separation within the TMD layer. However, the effect of the dipole-induced band bending on the photocurrent is likely limited, given the insignificant photoactivity improvement observed for the $\mathrm{MoSe}_{2} / \mathrm{DHA}-\mathrm{PDI}$ hybrid electrode despite the similar magnitude of band banding shown by XPS (0.4 and $0.3 \mathrm{eV}$ for $\mathrm{MoS}_{2}$ and $\mathrm{MoSe}_{2}$, respectively). Because the improvement in $J_{\mathrm{ph}}$ is considerably smaller than that using $\mathrm{MoS}_{2}$, we can also conclusively eliminate the possibility that the observed improvement of the $\mathrm{MoS}_{2}$ hybrid electrodes was only a result of defect passivation or surface polarization. Instead, the distinct photoelectrochemical performance can be attributed to a difference in band alignment. Indeed, the red-shifted excitonic energy in the UV-vis spectrum (Figure S11) of $\mathrm{MoSe}_{2}$ compared to that of $\mathrm{MoS}_{2}$ suggests a narrower direct band 
gap. Moreover, the previously mentioned higher-lying valence band level in $\mathrm{MoSe}_{2}$ gives a plausible explanation for the difference, shown schematically in Figure 5, where band structures for both $\mathrm{MoS}_{2}$ and $\mathrm{MoSe}_{2}$ hybrid interfaces are suggested. a) vac

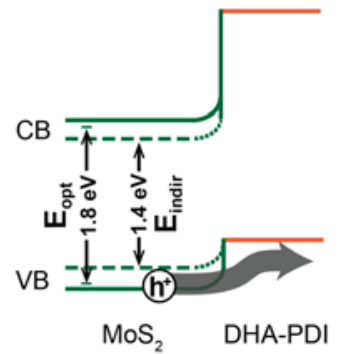

b)
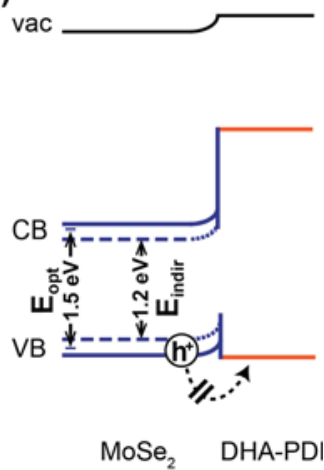

Figure 5. Schematic band alignment and hole transfer process in (a) $\mathrm{MoS}_{2}$ /DHA-PDI and (b) $\mathrm{MoSe}_{2}$ /DHA-PDI hybrid electrodes. In the TMD layers, solid lines represent the direct band edges, while the broken lines represent the indirect band edges. The optical band gap energy based on the excitonic absorption onset energy is also indicated.

For $\mathrm{MoSe}_{2}$, the higher-lying valence band edge likely results in a barrier for hole transfer at the interface between $\mathrm{MoSe}_{2}$ and DHA-PDI. Indeed, the identical excited-state dynamics in $\mathrm{MoSe}_{2}$ with and without an overlayer shown by the TA measurement support the conclusion that the DHA-PDI overlayer does not promote charge separation in $\mathrm{MoSe}_{2}$. The small increase in $J_{\mathrm{ph}}$ observed in the $\mathrm{MoSe}_{2} / \mathrm{DHA}-\mathrm{PDI}$ film can be attributed to the following: (1) light absorption by DHAPDI and electron injection into the substrates (either directly or through the conduction band of $\mathrm{MoSe}_{2}$ ), (2) reduced recombination at the exposed substrate/electrolyte interface given by the decreased dark current of the $\mathrm{MoSe}_{2}$ hybrid electrode, or (3) the interfacial dipole created at the $\mathrm{MoSe}_{2} /$ DHA-PDI interface providing a slight advantage for charge extraction directly from the $\mathrm{MoSe}_{2}$ to the electrolyte. On the other hand, type-II heterojunction formation with $\mathrm{MoS}_{2}$ and DHA-PDI is likely afforded by the deeper-lying valence band of $\mathrm{MoS}_{2}$. Such a junction would favor the transfer of photogenerated holes in $\mathrm{MoS}_{2}$ to DHA-PDI, reducing charge carrier recombination and resulting in increased $J_{\mathrm{ph}}$. We note that, while we observed hole transfer from the direct excited states, we cannot exclude hole transfer from the indirect valence band states (as illustrated in Figure 5a).

With regards to the possible role of surface trapping states in the different behavior of the hybrid heterojunctions, given the defect-poor planar surface of solvent-assisted exfoliated TMDs flakes, ${ }^{33}$ it is unlikely that trap states on the basal plane surface play a role. The density and energy of surface states at the flake edges are however important to consider. Indeed, the edge sites, where transition metal atoms are exposed, are known to be trap sites that cause severe recombination in TMDs. ${ }^{34} \mathrm{We}$ note that the morphology of $\mathrm{MoSe}_{2}$ films is strikingly similar to that of $\mathrm{MoS}_{2}$ (see Figure S12, Supporting Information). Given also the similar film thickness judged by UV-vis spectra, the density of the edge sites for $\mathrm{MoS}_{2}$ and $\mathrm{MoSe}_{2}$ is likely similar. While we cannot completely eliminate the possibility that photogenerated charge transfer is mediated by edge sites in the $\mathrm{MoS}_{2}$ /DHA-PDI film, whereas this does not occur in the $\mathrm{MoSe}_{2}$ /DHA-PDI film, we note that DHA-PDI does not primarily deposit on the TMD edges in the hybrid films (rather, we observed coating of the basal plane, as shown in Figure S4). With these observations, we consider the possibility that photocurrent enhancement via trap (edge)-assisted charge transfer is unlikely to be the principal mechanism for observed results. However, the role of the trap states is a point for further investigation in future work.

In summary, we exclusively use solution-based fabrication to prepare hybrid thin films consisting of $2 \mathrm{D}$ semiconducting TMD flakes and a functionalized PDI. Investigations of $\mathrm{MoS}_{2}-$ and $\mathrm{MoSe}_{2}$-based hybrid electrodes reveal interfacial dipole formation at the TMD/organic interface; however, this alone is insufficient to afford increased charge extraction from the TMD flakes. On the other hand, our results suggest that proper band alignment at the TMD-based inorganic-organic heterojunction is crucial to afford efficient charge separation and improved photon harvesting. This demonstration, for the first time, presents evidence for efficient charge separation (quantum efficiency up to $37 \%$ ) from liquid-exfoliated semiconducting 2D $\mathrm{MoS}_{2}$ at a hybrid heterojunction with an OS and suggests possibilities for new applications of semiconducting 2D TMDs in inorganic-organic hybrid systems. While the quantum efficiency of the hybrid films reported here is not far from that of other optimized colloidal nanoparticle/OS hybrid heterojunctions (which typically give quantum efficiency in the 40$50 \%$ range $^{35}$ ), the performance is certainly inferior to singlecrystal-based solar energy conversion devices or even optimized bulk heterojunction organic photovoltaics. However, facile preparation of the ultrathin TMD-based devices by solutionbased processing shown herein and the known robustness of TMDs make these materials attractive candidates for inexpensive, large-area, and stable solar energy conversion devices. Moreover, the hybrid approach provides advantages such as facile functionalization and energy level tuning of the OSs, which allow alternate routes for device performance optimization and the possibility to employ these heterojunctions in diverse applications. For example, a functional group on the OS could graft a water oxidation catalyst, potentially enabling $\mathrm{MoS}_{2}$ as a light absorber for the solar oxygen evolution reaction. We note that further optimization of the layer thicknesses and study of the defects, edge sites, and the effect of the orientation of the OS at the heterojunction interface will likely lead to insight into the limitations of charge transfer and recombination. These are points of interest for future work to improve the performance of these systems. Overall, the continued development of TMD-organic hybrid heterojunction-based devices, together with our demonstration of the facile solution-based processing, will make these hybrid systems promising for global-scale solar energy conversion.

\section{ASSOCIATED CONTENT}

\section{S Supporting Information}

The Supporting Information is available free of charge on the ACS Publications website at DOI: 10.1021/acsenergylett.6b00707.

Detailed methods regarding electrode fabrication and characterization setup and parameters, PDI derivatives' synthesis and characterization, additional electron microscopy and morphology analysis of $\mathrm{MoS}_{2}$ and 
hybrid electrodes, Raman spectra, two-electrode cells, J$V$ curves for control experiments, additional XPS spectra, UV-vis absorption spectra for $\mathrm{MoSe}_{2}$-based films, additional TA spectra, and detailed TA spectra interpretation (PDF)

\section{AUTHOR INFORMATION}

\section{Corresponding Author}

*E-mail: kevin.sivula@epfl.ch.

\section{ORCID 우}

Natalie Banerji: 0000-0001-9181-2642

Kevin Sivula: 0000-0002-8458-0270

\section{Notes}

The authors declare no competing financial interest.

\section{ACKNOWLEDGMENTS}

This research was supporting in part by the Swiss Competence Centers for Energy Research (SCCER Heat and Electricity Storage, Contract \# KTI.2014.0113) and the European Research Commission (ERC starting grant "CEMOS” Project 336506).

\section{REFERENCES}

(1) Aruchamy, A. Photoelectrochemistry and Photovoltaics of Layered Semiconductors; Springer: The Netherlands, 1992.

(2) Yu, X.; Sivula, K. Toward Large-Area Solar Energy Conversion with Semiconducting 2D Transition Metal Dichalcogenides. ACS Energy Lett. 2016, 1, 315-322.

(3) Britnell, L.; Ribeiro, R. M.; Eckmann, A.; Jalil, R.; Belle, B. D.; Mishchenko, A.; Kim, Y.-J.; Gorbachev, R. V.; Georgiou, T.; Morozov, S. V.; et al. Strong Light-Matter Interactions in Heterostructures of Atomically Thin Films. Science 2013, 340, 1311-1314.

(4) Wang, Q. H.; Kalantar-Zadeh, K.; Kis, A.; Coleman, J. N.; Strano, M. S. Electronics and Optoelectronics of Two-dimensional Transition Metal Dichalcogenides. Nat. Nanotechnol. 2012, 7, 699-712.

(5) Haigh, S. J.; Gholinia, A.; Jalil, R.; Romani, S.; Britnell, L.; Elias, D. C.; Novoselov, K. S.; Ponomarenko, L. A.; Geim, A. K.; Gorbachev, R. Cross-sectional Imaging of Individual Layers and Buried Interfaces of Graphene-based Heterostructures and Superlattices. Nat. Mater. 2012, 11, 764-767.

(6) Jariwala, D.; Marks, T. J.; Hersam, M. C. Mixed-dimensional van der Waals Heterostructures. Nat. Mater. 2016, 16, 170-181.

(7) Geim, A. K.; Grigorieva, I. V. Van der Waals Heterostructures. Nature 2013, 499, 419-425.

(8) Liu, F.; Chow, W. L.; He, X.; Hu, P.; Zheng, S.; Wang, X.; Zhou, J.; Fu, Q.; Fu, W.; Yu, P.; et al. Van der Waals p-n Junction Based on an Organic-Inorganic Heterostructure. Adv. Funct. Mater. 2015, 25, 5865-5871.

(9) Jariwala, D.; Howell, S. L.; Chen, K.-S.; Kang, J.; Sangwan, V. K.; Filippone, S. A.; Turrisi, R.; Marks, T. J.; Lauhon, L. J.; Hersam, M. C. Hybrid, Gate-Tunable, van der Waals $\mathrm{p}-\mathrm{n}$ Heterojunctions from Pentacene and $\mathrm{MoS}_{2}$. Nano Lett. 2016, 16, 497-503.

(10) Zhao, M.; Chang, M.-J.; Wang, Q.; Zhu, Z.-T.; Zhai, X.-P.; Zirak, M.; Moshfegh, A. Z.; Song, Y.-L.; Zhang, H.-L. Unexpected optical limiting properties from $\mathrm{MoS}_{2}$ nanosheets modified by a semiconductive polymer. Chem. Commun. 2015, 51, 12262-12265.

(11) Coleman, J. N.; Lotya, M.; O’Neill, A.; Bergin, S. D.; King, P. J.; Khan, U.; Young, K.; Gaucher, A.; De, S.; Smith, R. J.; et al. TwoDimensional Nanosheets Produced by Liquid Exfoliation of Layered Materials. Science 2011, 331, 568-571.

(12) Yu, X.; Prevot, M. S.; Guijarro, N.; Sivula, K. Self-assembled 2D $\mathrm{WSe}_{2}$ Thin Films for Photoelectrochemical Hydrogen Production. Nat. Commun. 2015, 6, 7596.
(13) Zhan, X.; Facchetti, A.; Barlow, S.; Marks, T. J.; Ratner, M. A.; Wasielewski, M. R.; Marder, S. R. Rylene and Related Diimides for Organic Electronics. Adv. Mater. 2011, 23, 268-284.

(14) Kaneda, Y.; Stawasz, M. E.; Sampson, D. L.; Parkinson, B. A. STM Investigations of the Two-Dimensional Ordering of Perylenetetracarboxylic Acid N-Alkyl-diimides on HOPG and $\mathrm{MoS}_{2}$ Surfaces. Langmuir 2001, 17, 6185-6195.

(15) Würthner, F.; Saha-Möller, C. R.; Fimmel, B.; Ogi, S.; Leowanawat, P.; Schmidt, D. Perylene Bisimide Dye Assemblies as Archetype Functional Supramolecular Materials. Chem. Rev. 2016, 116, 962-1052.

(16) Gong, Y.; Lin, J.; Wang, X.; Shi, G.; Lei, S.; Lin, Z.; Zou, X.; Ye, G.; Vajtai, R.; Yakobson, B. I.; et al. Vertical and in-plane heterostructures from $\mathrm{WS}_{2} / \mathrm{MoS}_{2}$ monolayers. Nat. Mater. 2014, 13, $1135-1142$.

(17) Tongay, S.; Fan, W.; Kang, J.; Park, J.; Koldemir, U.; Suh, J.; Narang, D. S.; Liu, K.; Ji, J.; Li, J.; et al. Tuning Interlayer Coupling in Large-Area Heterostructures with CVD-Grown $\mathrm{MoS}_{2}$ and $\mathrm{WS}_{2}$ Monolayers. Nano Lett. 2014, 14, 3185-90.

(18) Bernardi, M.; Palummo, M.; Grossman, J. C. Extraordinary Sunlight Absorption and One Nanometer Thick Photovoltaics Using Two-Dimensional Monolayer Materials. Nano Lett. 2013, 13, 36643670.

(19) Beal, A. R.; Hughes, H. P. Kramers-Kronig Analysis of the Reflectivity Spectra of $2 \mathrm{H}-\mathrm{MoS}_{2}, 2 \mathrm{H}-\mathrm{MoSe}_{2}$ and $2 \mathrm{H}-\mathrm{MoTe}_{2}$. J. Phys. C: Solid State Phys. 1979, 12, 881-890.

(20) King, L. A.; Zhao, W.; Chhowalla, M.; Riley, D. J.; Eda, G. Photoelectrochemical Properties of Chemically Exfoliated $\mathrm{MoS}_{2}$. J. Mater. Chem. A 2013, 1, 8935-8941.

(21) Xu, X.; Hu, J.; Yin, Z.; Xu, C. Photoanode Current of LargeArea $\mathrm{MoS}_{2}$ Ultrathin Nanosheets with Vertically Mesh-Shaped Structure on Indium Tin Oxide. ACS Appl. Mater. Interfaces 2014, 6, $5983-5987$.

(22) White, H. S.; Fan, F. R. F.; Bard, A. J. Semiconductor Electrodes: XXXIII. Photoelectrochemistry of n-Type $\mathrm{WSe}_{2}$ in Acetonitrile. J. Electrochem. Soc. 1981, 128, 1045-1055.

(23) Jakubowicz, A.; Mahalu, D.; Wolf, M.; Wold, A.; Tenne, R. $\mathrm{WSe}_{2}$ : Optical and Electrical Properties as Related to Surface Passivation of Recombination Centers. Phys. Rev. B: Condens. Matter Mater. Phys. 1989, 40, 2992-3000.

(24) Spadafora, E. J.; Demadrille, R.; Ratier, B.; Grévin, B. Imaging the Carrier Photogeneration in Nanoscale Phase Segregated Organic Heterojunctions by Kelvin Probe Force Microscopy. Nano Lett. 2010, $10,3337-3342$.

(25) Dumcenco, D.; Ovchinnikov, D.; Marinov, K.; Lazić, P.; Gibertini, M.; Marzari, N.; Sanchez, O. L.; Kung, Y.-C.; Krasnozhon, D.; Chen, M.-W.; et al. Large-Area Epitaxial Monolayer $\mathrm{MoS}_{2}$. ACS Nano 2015, 9, 4611-20.

(26) Lin, J. D.; Han, C.; Wang, F.; Wang, R.; Xiang, D.; Qin, S.; Zhang, X.-A.; Wang, L.; Zhang, H.; Wee, A. T. S.; et al. ElectronDoping-Enhanced Trion Formation in Monolayer Molybdenum Disulfide Functionalized with Cesium Carbonate. ACS Nano 2014, $8,5323-5329$.

(27) Tao, J.; Chai, J. W.; Zhang, Z.; Pan, J. S.; Wang, S. J. The Energy-band Alignment at Molybdenum Disulphide and High-k dielectrics Interfaces. Appl. Phys. Lett. 2014, 104, 232110.

(28) Haring, A. J.; Ahrenholtz, S. R.; Morris, A. J. Rethinking Band Bending at the $\mathrm{P} 3 \mathrm{HT}-\mathrm{TiO}_{2}$ Interface. ACS Appl. Mater. Interfaces 2014, 6, 4394-4401.

(29) Tsokkou, D.; Yu, X.; Sivula, K.; Banerji, N. The Role of Excitons and Free Charges in the Excited-State Dynamics of Solution-Processed Few-Layer $\mathrm{MoS}_{2}$ Nanoflakes. J. Phys. Chem. C 2016, 120, 2328623292.

(30) Kang, J.; Tongay, S.; Zhou, J.; Li, J.; Wu, J. Band Offsets and Heterostructures of Two-dimensional Semiconductors. Appl. Phys. Lett. 2013, 102, 012111.

(31) Rasmussen, F. A.; Thygesen, K. S. Computational 2D Materials Database: Electronic Structure of Transition Metal Dichalcogenides and Oxides. J. Phys. Chem. C 2015, 119, 13169-13183. 
(32) Ambrosi, A.; Sofer, Z.; Pumera, M. $2 \mathrm{H} \rightarrow 1 \mathrm{~T}$ Phase Tansition and Hydrogen Evolution Activity of $\mathrm{MoS}_{2}, \mathrm{MoSe}_{2}, \mathrm{WS}_{2}$ and $\mathrm{WSe}_{2}$ Strongly Depends on the $\mathrm{MX}_{2}$ Composition. Chem. Commun. 2015, $51,8450-3$.

(33) Backes, C.; Higgins, T. M.; Kelly, A.; Boland, C.; Harvey, A.; Hanlon, D.; Coleman, J. N. Guidelines for Exfoliation, Characterization and Processing of Layered Materials Produced by Liquid Exfoliation. Chem. Mater. 2017, 29, 243-255.

(34) Canfield, D.; Parkinson, B. A. Improvement of Energy Conversion Efficiency by Specific Chemical Treatments of Molybdenum Selenide ( $\left.\mathrm{n}-\mathrm{MoSe}_{2}\right)$ and Tungsten Selenide (n-WSe ${ }_{2}$ photoanodes. J. Am. Chem. Soc. 1981, 103, 1279-1281.

(35) Xu, T.; Qiao, Q. Conjugated Polymer-inorganic Semiconductor Hybrid Solar Cells. Energy Environ. Energy Environ. Sci. 2011, 4, $2700-2720$ 